
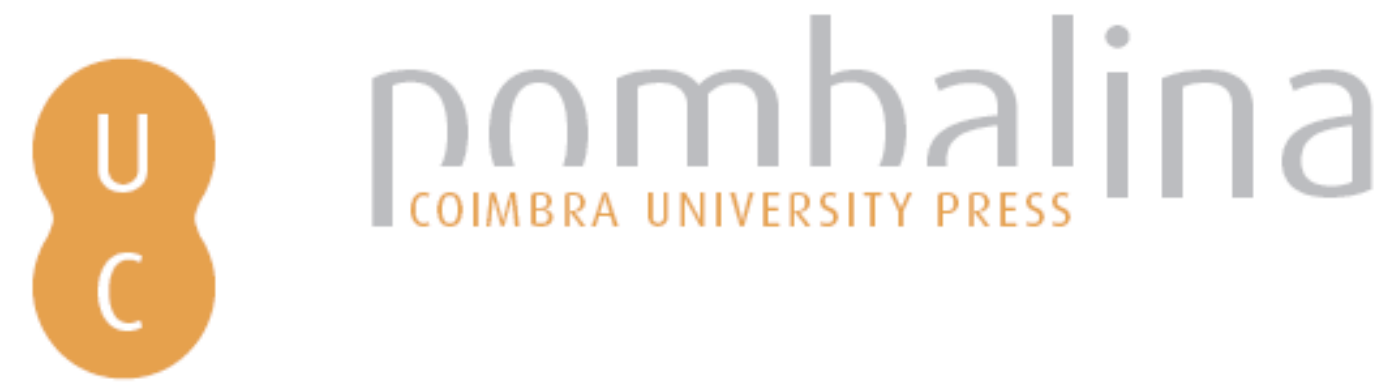

\title{
Avaliação do risco de galgamentos e inundação: integração de um método expedito de inundação no sistema hidralerta
}
Autor(es):
Poseiro, P.; Fortes, C. J. E. M.; Reis, M. T.
Publicado por: Imprensa da Universidade de Coimbra; RISCOS - Associação
URL persistente:
Portuguesa de Riscos, Prevenção e Segurança
DOI:
URI:http://hdl.handle.net/10316.2/34874
DOI:http://dx.doi.org/10.14195/978-989-96253-3-4_73
Accessed : $\quad$ 7-Jan-2015 12:43:50

A navegação consulta e descarregamento dos títulos inseridos nas Bibliotecas Digitais UC Digitalis, UC Pombalina e UC Impactum, pressupõem a aceitação plena e sem reservas dos Termos e Condições de Uso destas Bibliotecas Digitais, disponíveis em https://digitalis.uc.pt/pt-pt/termos.

Conforme exposto nos referidos Termos e Condições de Uso, o descarregamento de títulos de acesso restrito requer uma licença válida de autorização devendo o utilizador aceder ao(s) documento(s) a partir de um endereço de IP da instituição detentora da supramencionada licença.

Ao utilizador é apenas permitido o descarregamento para uso pessoal, pelo que o emprego do(s) título(s) descarregado(s) para outro fim, designadamente comercial, carece de autorização do respetivo autor ou editor da obra.

Na medida em que todas as obras da UC Digitalis se encontram protegidas pelo Código do Direito de Autor e Direitos Conexos e demais legislação aplicável, toda a cópia, parcial ou total, deste documento, nos casos em que é legalmente admitida, deverá conter ou fazer-se acompanhar por este aviso.

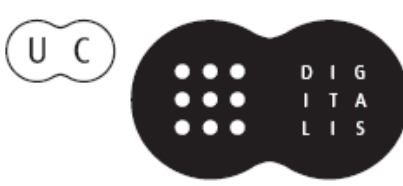



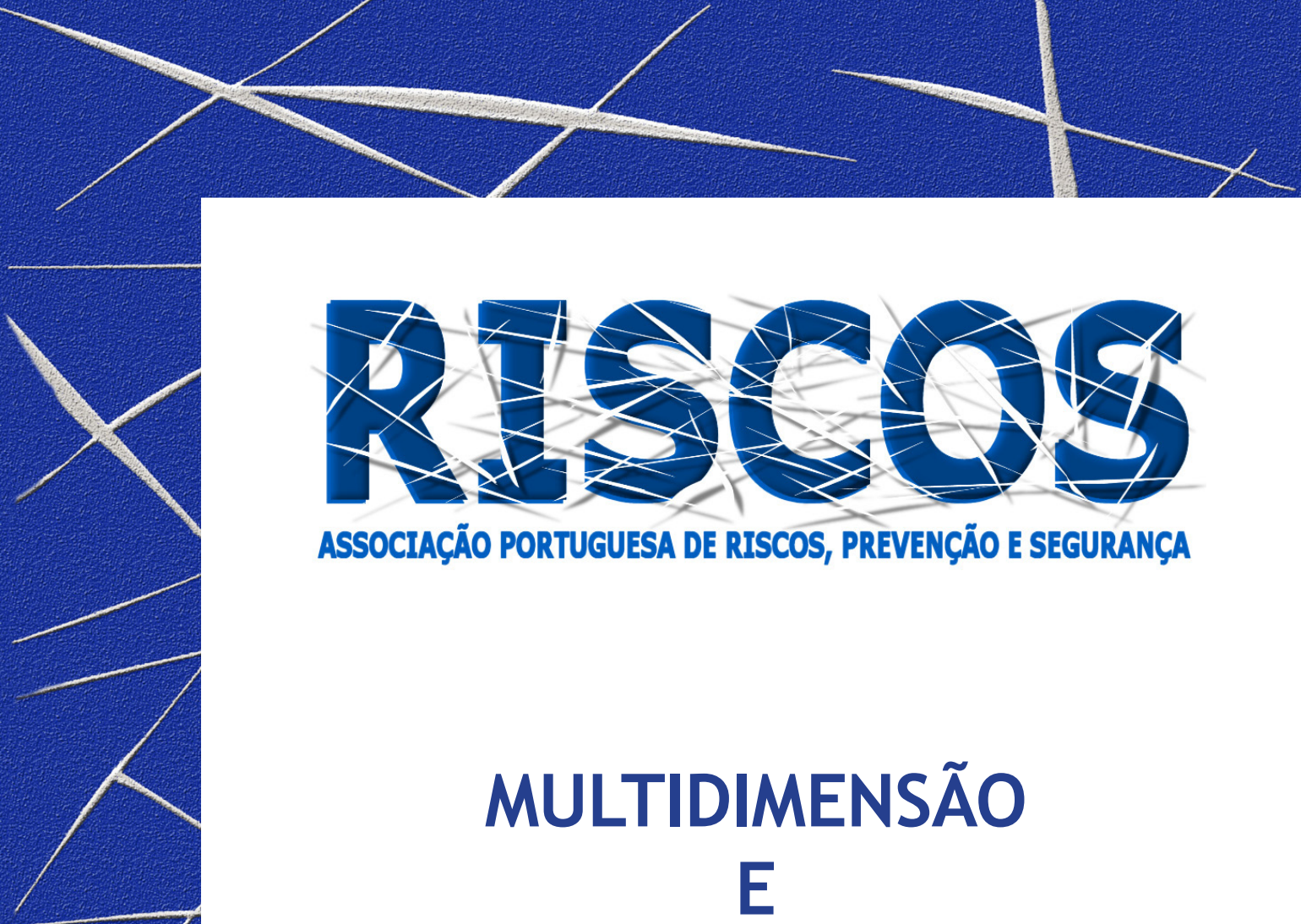

ASSOCIAÇÃO PORTUGUESA DE RISCOS, PREVENCCÃO E SEGURANÇA

MULTIDIMENSÃO

E
TERRITÓRIOS DE RISCO

III Congresso Internacional

I Simpósio Ibero-Americano

VIII Encontro Nacional de Riscos

Guimarães

2014 


\title{
AVALIAÇÃO DO RISCO DE GALGAMENTOS E INUNDAÇÃO: INTEGRAÇÃO DE UM MÉTODO EXPEDITO DE INUNDAÇÃO NO SISTEMA HIDRALERTA
}

\author{
P. Poseiro, C. J. E. M. Fortes, M. T. Reis \\ Laboratório Nacional de Engenharia Civil (LNEC) \\ pposeiro@lnec.pt, jfortes@lnec.pt, treis@lnec.pt

\section{RESUMO}

O HIDRALERTA é um sistema de previsão, alerta e avaliação do risco associado aos galgamentos e inundação em zonas portuárias, tendo como ideia-base a utilização de medições/previsões da agitação marítima para calcular o galgamento e a inundação nessas zonas. A avaliação do risco associado a estes fenómenos é efetuada através da definição de limiares para os caudais galgados ou para as cotas de inundação admissíveis, e pelo produto do grau da probabilidade de ocorrência de valores desses caudais pelo grau das consequências desses acontecimentos.

No sistema, o cálculo dos caudais médios galgados sobre uma estrutura é efetuado com recurso a ferramentas neuronais (NN_OVERTOPPING2) e/ou ferramentas empíricas, que são métodos expeditos e que não fornecem indicação sobre a distribuição espacial desse caudal na zona atrás da estrutura.

Nesta comunicação, é apresentada uma metodologia incorporada no HIDRALERTA com o intuito de modelar o galgamento e a inundação na sua distribuição espacial, recorrendo a ferramentas SIG. Essa metodologia consiste na utilização dos resultados da NN_OVERTOPPING2 para o cálculo do caudal médio galgado por metro linear de estrutura, a partir de onde se pode obter o volume de água correspondente e posterior distribuição desse volume no modelo Digital do Terreno da zona em estudo. 0 caso de estudo localiza-se no porto e baia da Praia da Vitória, na Itha Terceira, nos Açores.

Palavras-chave: HIDRALERTA; Agitação marítima; Galgamento; Inundação; Risco.

\section{Zona de estudo - Porto e baía da Praia da Vitória}

O porto e a baía da Praia da Vitória localizam-se na costa Este da ltha Terceira, uma das nove ilhas do arquipélago dos Açores, Portugal (Figura 1a). A Praia da Vitória é limitada a norte pela Ponta da Má Merenda e a sul pela Ponta do Espírito Santo e tem cerca de 2400 m de comprimento e $1100 \mathrm{~m}$ de largura máxima. 0 porto é protegido por dois quebra-mares (sul e norte) e a linha de costa da baía caracteriza-se pela existência de uma defesa frontal aderente com cerca de 1 $\mathrm{km}$ de comprimento e de um campo de cinco esporões na zona central que enraízam na defesa frontal. 
CAPÍTULO 3.2: RISCOS CLIMÁTICOS E HIDROLÓGICOS
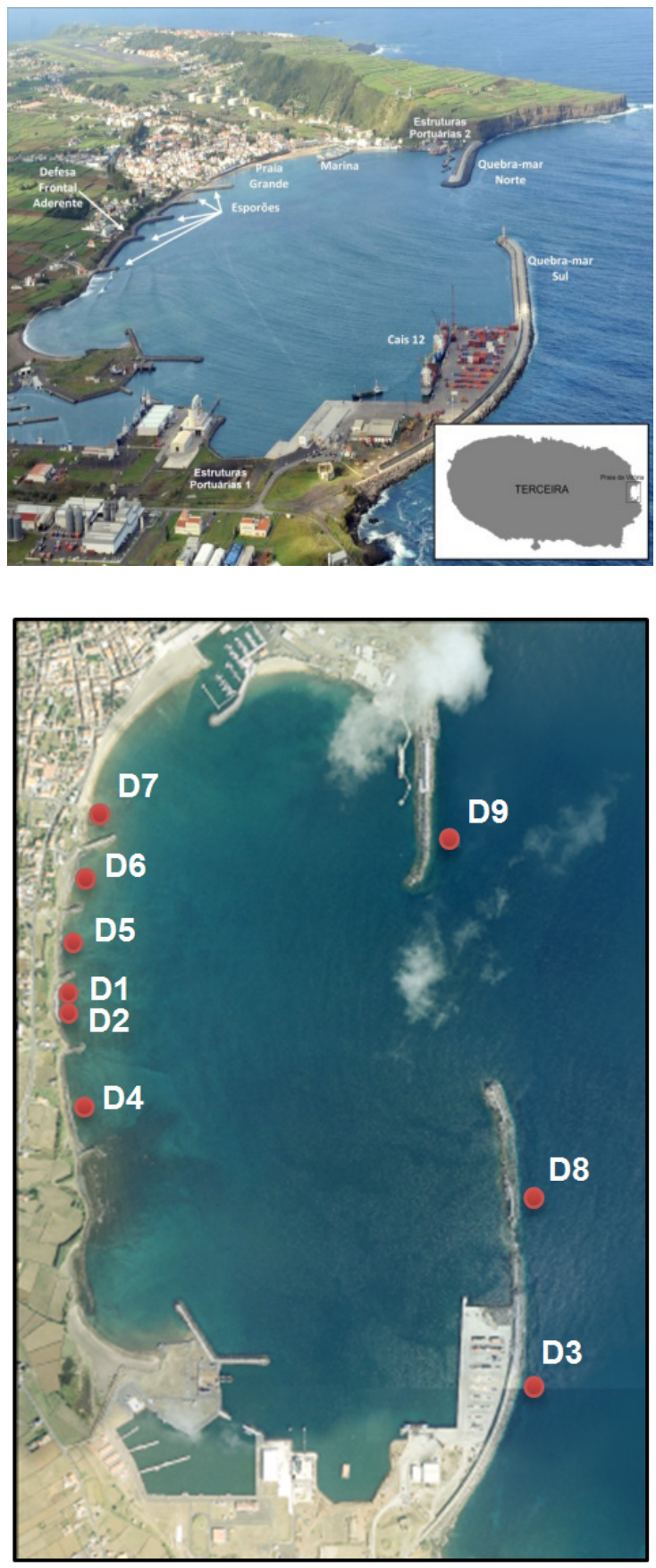

Figura 1. a) Porto da Praia da Vitória: b) Secções estudadas (D1 a D9) 
No âmbito do projeto HIDRLERTA em curso (Fortes et al., 2014), e para este porto, encontra-se em desenvolvimento um protótipo de um sistema de previsão e alerta de risco associado ao galgamento e inundação em zonas costeiras e portuárias, o sistema HIDRALERTA (Poseiro et al., 2013a, Poseiro et al., 2013b; Fortes et al., 2013; Fortes et al., 2014). O sistema está a ser desenvolvido em linguagem de programação Python e implementado numa plataforma WebSIG. A metodologia do sistema consiste em:

- Definir o regime de agitação marítima incidente nas estruturas portuárias recorrendo a dados obtidos in situ e/ou a resultados de modelos numéricos de propagação de ondas;

- Calcular a resposta da estrutura em termos de caudal médio galgado por unidade de comprimento do coroamento da estrutura, com a utilização da ferramenta neuronal NN_OVERTOPPING2 (Coeveld, 2005);

- Calcular o grau de risco associado à ocorrência de galgamentos acima de um determinado caudal médio pré-definido para cada estrutura, em função do tipo de estrutura galgada e das atividades e bens por ela protegidos. A avaliação do risco associado a estes fenómenos é efetuada através da definição de limiares para os caudais galgados ou para as cotas de inundação admissíveis, e pelo produto do grau da probabilidade de ocorrência de valores desses caudais pelo grau das consequências desses acontecimentos.

Em trabalhos anteriores, foi efetuada uma avaliação do risco para nove estruturas do porto (Figura 1b), para um período de tempo de 5 anos (Poseiro et al., 2013a; Reis et al. 2014). No entanto, uma vez que se utilizou a ferramenta neuronal NN_OVERTOPPING2 para a determinação do caudal, esta não fornece indicação sobre a distribuição espacial desse caudal atrás da estrutura. Desta forma, o presente trabalho é uma extensão de trabalhos anteriores, e apresenta uma metodologia para modelar o galgamento e a inundação na sua distribuição espacial recorrendo a ferramenta GIS. Para este efeito, pretende-se converter os caudais obtidos pela NN_OVERTOPPING2 em volumes e estabelecer a relação entre os volumes e a cota de inundação obtida para cada secção com a utilização de um Modelo Digital do Terreno (MDT) da zona em estudo. Uma vez conhecidas as áreas inundáveis e juntamente com a metodologia já desenvolvida para obter o mapa de consequências através da aplicação do Processo de Análise Hierárquica (AHP) (Poseiro et al., 2013b), é possível obter o mapa de risco, tendo em conta simultaneamente as áreas mais facilmente galgadas/inundadas e o peso das atividades que se desenvolvem em cada uma dessas áreas.

\section{Metodologia para o cálculo da área inundável}

A determinação do regime de agitação incidente no ponto D4 (Figura 1b) é efetuada utilizando os resultados das previsões da agitação marítima ao largo da zona considerada para o período correspondente aos anos de 2008 a 2012, obtidos com o modelo WAVEWATCH III (Tolman, 1999). Esses resultados são transferidos para o interior da baía e do porto com recurso a dois modelos de propagação e deformação da agitação marítima: o modelo SWAN (Booj, 1999) e o modelo DREAMS (Fortes, 1993). Uma vez conhecidos esses valores, e para as características da estrutura selecionada (D4), aplicou-se a ferramenta neuronal NN_OVERTOPPING2 e efetuou-se o cálculo do caudal médio galgado (Figura 2). 


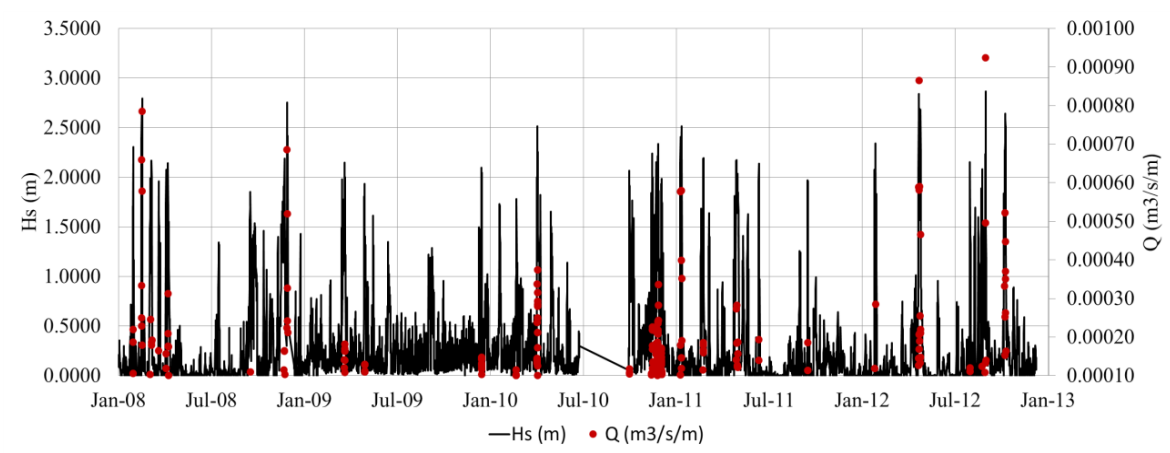

Figura 2. Altura Significativa da onda e Caudal médio galgado na secção D4

Para a avaliação da área inundável associada a um dado valor de caudal médio galgado por metro linear de estrutura $\left(\mathrm{m}^{3} / \mathrm{s} / \mathrm{m}\right)$, é necessário primeiro converter estas unidades para volumes. Uma vez que a disponibilização dos resultados da agitação marítima é feita de 6 em 6 horas, fez-se o produto entre os resultados da rede neuronal e $21600 \mathrm{~s}$ (6h) e desta forma, obtiveram-se os resultados com as seguintes unidades: $\mathrm{m}^{3} / \mathrm{m}$ de estrutura. Com vista a fazer a sua distribuição espacial considerou-se o modelo Digital de Terreno da zona onde esse caudal vai atuar (Figura 3a). Na Figura 3b) são apresentadas as características do local atrás da estrutura da secção D4.

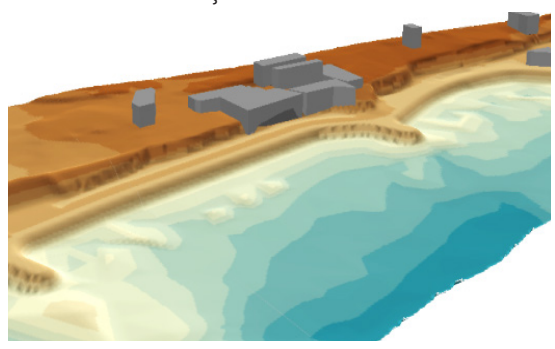

a)

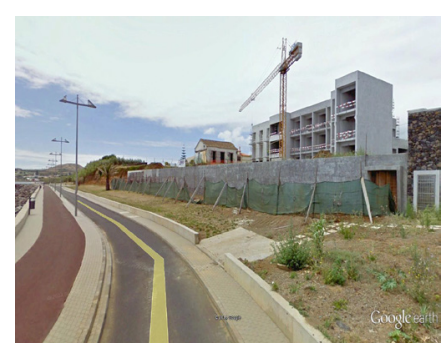

b)

Figura 3. a) Modelo Digital do Terreno do porto da Praia da Vitória. b) Fotografia com as características do local atrás da estrutura da secção D4.

Para determinar valores pré-definidos da cota de área inundável, considerou-se uma secção com a largura de 1 metro (Figura 4a) e determinou-se o volume correspondente para diferentes cotas de inundação, com recurso à ferramenta Surface Volume do ArcGIS 10.2 ${ }^{\mathrm{TM}}$. Desta forma, foi possível estabelecer uma função polinomial de $2^{\circ}$ grau ajustada aos valores obtidos com um coeficiente de determinação $\left(\mathrm{R}^{2}\right)$ de 0.9841 (Figura $4 \mathrm{~b}$ ). 

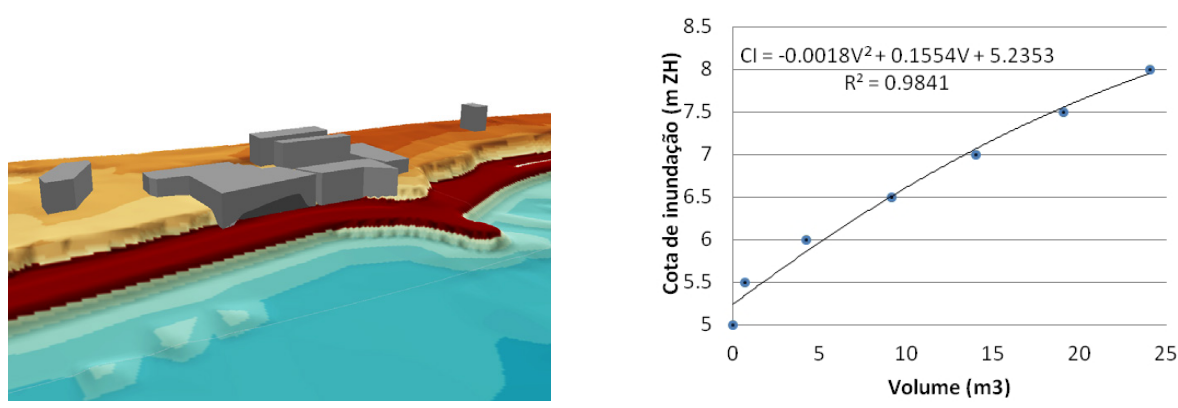

Figura 4. a) Secção considerada para medir a relação entre as cotas de inundação e os volumes. b) Relação entre o volume da área inundável e a altura.

Para os cinco anos de dados (de 2008 a 2012) obtiveram-se os resultados da Tabela I).

Tabela I - Resultados dos galgamentos, volumes e cotas de inundação obtidos para a secção D4 durante 5 anos (2008 a 2012).

\begin{tabular}{|c|c|c|c|c|}
\hline & Quant. (6h) & Min & Max & Média \\
\hline \multirow{2}{*}{$\mathrm{Q}\left(\mathrm{m}^{3} / \mathrm{s} / \mathrm{m}\right)$} & \multirow{3}{*}{320} & 0.00005 & 0.00092 & 0.00016 \\
\cline { 1 - 3 } $\mathrm{V}\left(\mathrm{m}^{3} / \mathrm{m}\right)$ & & 1.07 & 19.95 & 3.52 \\
\cline { 1 - 3 } $\mathrm{nnyyy}$ & & 5.42 & 7.61 & 5.82 \\
\hline
\end{tabular}

Na Figura 5 está representada (a vermelho) a cota máxima de inundação para a secção D4.

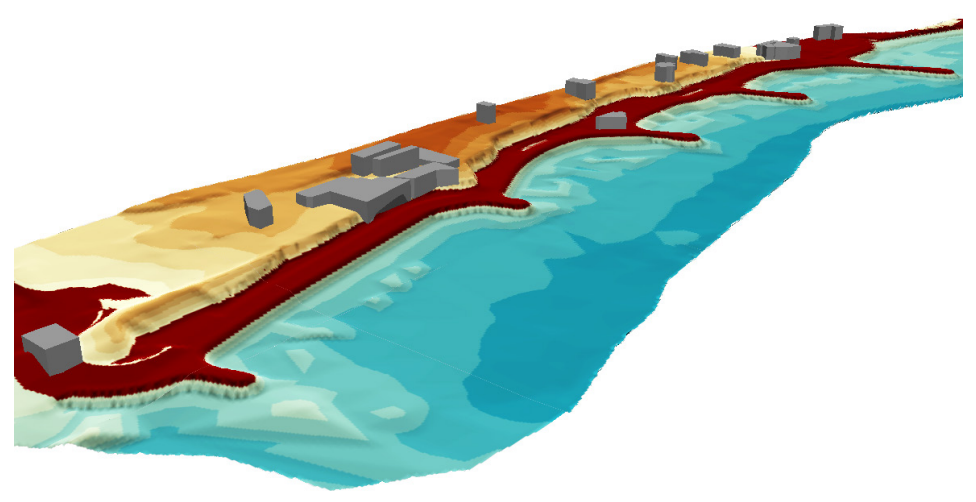

Figura 5. Cota máxima de inundação regista para os 5 anos em análise

É possível verificar na Figura 5 que os resultados máximos registados colocam em causa não só as atividades que decorram atrás da defesa, que incluem as pessoas (passadeira) e as viaturas (estrada), mas também o edifício localizado nas imediações. 


\section{Conclusão}

Esta comunicação descreve uma metodologia incorporada no sistema HIDRALERTA com o intuito de modelar o galgamento e a inundação na sua distribuição espacial, recorrendo a ferramentas SIG. Essa metodologia consiste na integração dos resultados da NN_OVERTOPPING2 com um modelo Digital do Terreno da zona em estudo.

A aplicação à Praia da Vitória e, mais especificamente, à estrutura aderente entre o 4 e 5 esporão (D4) mostrou que a metodologia é viável e é uma forma expedita de cálculo da área inundável. No entanto, é uma metodologia aplicada por seção linear da estrutura admitindo assim a constância das condições para toda a secção D4.

No futuro a utilização de modelos numéricos de inundação constituirão ferramentas mais poderosas para a definição das áreas inundáveis. Por outro lado, também a disponibilização dos resultados com maior frequência, de 1 em 1 hora, permitirá a obtenção de resultados mais fidedignos. Será necessário proceder também à validação destes resultados e proceder à sua aplicação para as restantes secções para que finalmente se possa fazer o cruzamento desta informação com o mapa de consequências com vista a obter um mapa de risco.

\section{Bibliografia}

Booj, N., Ris, R., Holthuijsen, L., 1999. A third-generation wave model for coastal regions. Part I, Model description and validation. Journal of Geographical Research, C4(104), pp. 7649-7666.

Coeveld, E.M., Van Gent, M.R.A. e Pozueta, B. (2005). Neural Network Manual NN_OVERTOPPING2.CLASH WP8, June.

Fortes, C.J.E.M. (1993). Modelação matemática da refração e difração combinadas de ondas marítimas (análise pelo método dos elementos finitos). Tese de Mestrado em Engenharia Mecânica, IST, Lisboa, Portugal.

Fortes, C.J.E.M., Reis, M.T., Poseiro, P., Capitão, R., Santos, J.A., Pinheiro, L.P., Rodrigues, A., Sabino, A., Rodrigues, M.C., Raposeiro, P., Ferreira, J.C., Silva, C., Simões, A., Azevedo, E.B. (2013). O Projeto HIDRALERTA. Sistema de Previsão e Alerta de Inundações em Zonas Costeiras e Portuárias. $8^{\mathrm{a}} \mathrm{s}$ JPECP, LNEC, 10 e 11 de outubro.

Fortes, C.J.E.M., Reis, M.T., Poseiro, P., Santos, J.A., Capitão, R., Pinheiro, L., Sabino; A., Rodrigues, A.; Ferreira, J.C., Martinho, S., Raposeiro, P., SILVA, C., Simões, A., Azevedo, E.B., Rodrigues M.C. (2014) Desenvolvimentos recentes do HIDRALERTA - Sistema de previsão e alerta de inundações em zonas costeiras e portuárias. 3as Jornadas de Engenharia Geográfica, IH, 24 a 26 de junho.

Poseiro, P., Fortes, C.J.E.M., Reis, M.T., Santos, J.A., Simões, A., Rodrigues, C. e Azevedo, E. (2013a). A methodology for overtopping risk assessment in port areas: Application to the Port of Praia da Vitória (Azores, Portugal). Proc. SCACR 2013, 4 a 7 de junho, LNEC, Portugal.

Poseiro, P., Fortes, C.J.E.M., Santos, J.A., Reis, M.T. e Craveiro, J. (2013b). Aplicação do processo de análise hierárquica (AHP) à análise das consequências de ocorrência de galgamentos. 0 caso da baía da Praia da Vitória. $8^{\text {a }}$ S JPECP, 10-11 outubro.

Reis, R. Fortes, C.J.E.M., Reis, M.T., Poseiro, P., Santos, J.A. (2014). "Avaliação comparativa de ferramentas neuronais. Aplicação à Praia da Vitória, Terceira, Açores”. VI SEMENGO - Seminário e Workshop em Engenharia Oceânica, Rio Grande, RS-Brasil, 12-14 novembro (Submetido).

Tolman, H. (1999). User Manual and System Documentation of WAVEWATCH-III. version 1.18, NOA/NWS/ NCEP/OMB Technical Note 166, 110p., Washington, E.U.A. 